\title{
Multi-objective machining parameter optimisation for residual stress based on quantum cat swarm
}

\section{Guohai Zhang}

School of Agricultural Engineering and Food Science,

Shandong University of Technology,

Zibo 255000, Shangdong, China

Email: guohaizhang@163.com

\section{Huibin Sun*}

Key Laboratory of Contemporary Design and Integrated Manufacturing Technology,

Ministry of Education,

Northwestern Polytechnical University,

Xi'an, Shaanxi, 710072, China

Email: sun_huibin@nwpu.edu.cn

*Corresponding author

\begin{abstract}
Residual stresses greatly affect parts' performances, lives, fatigue strengths, corrosion resistance, etc. Due to the lack of analytical models, machining parameter optimisation for better residual stresses is still a problem. In this paper, a multi-objective machining parameter optimisation method is proposed. Based on the support vector machine, the machining parameters' nonlinear relationships with the surface roughness and the residual stress are investigated. The cutting time consumption, surface roughness and absolute residual stress are the objectives, while the cutting speed, feed rate, axial cutting depth and the radial cutting deep are variables. The cutting power and cutting torque are constraints. The multi-objective cat swarm optimisation is designed to solve the problem, while the quantum computation is used to improve its performance. An experimental study is presented to verify the method. Some Pareto solutions are obtained with good convergence and diversity. Compared with the empirical machining parameters, the material removal rate, surface roughness and residual stress are optimised greatly. Compared with non-dominated sorting genetic algorithm II, the algorithm's precision and effectiveness are also verified.
\end{abstract}

Keywords: residual stress; machining parameter optimisation; multi-objective optimisation; cat swarm optimisation; quantum computation.

Reference to this paper should be made as follows: Zhang, G. and Sun, H. (2017) 'Multi-objective machining parameter optimisation for residual stress based on quantum cat swarm', Int. J. Service and Computing Oriented Manufacturing, Vol. 3, No. 1, pp.54-70.

Biographical notes: Guohai Zhang is an Associate Professor in Shandong University of Technology. He received his $\mathrm{PhD}$ in Mechanical Engineering from Xi'an Jiaotong University, Xi'an, China, in 2007. His research interests include iPSS, digital manufacturing, intelligent manufacturing, and so on. 
Huibin Sun is an Associate Professor in Northwestern Polytechnical University. $\mathrm{He}$ received his $\mathrm{PhD}$ in Mechanical Engineering from Xi'an Jiaotong University, Xi'an, China, in 2007. His research interests include iPSS, cutting tool wear condition monitoring and remaining useful life prediction, etc.

\section{Introduction}

Inevitably, residual stresses always remain in machined parts. Undesirable residual stresses dramatically affect parts' performances, lives, fatigue strengths, corrosion resistance, etc. (Li and Wang, 2017). Then, great efforts should be made to control residual stresses. Optimising machining parameter is a feasible option (Masoudi et al., 2015). In fact, machining parameter optimisation for better residual stresses is facing the following barriers.

1 The relationship between machining parameters and residual stresses is so complex. No analytical model is available to precisely decide machining parameters' effects on residual stresses (Masoudi et al., 2015). Then, how to build the relationship is a problem that must be solved prior to optimisation. Machining parameters based on handbooks or operators' experience are arbitrary or not optimised. Due to the lack of analytical models, machining parameters optimised by the empirical formulas are not reliable enough (Zhang et al., 2009).

2 Machining parameter optimisation is a typical multi-objective optimisation problem (MOP) when two or more objectives are considered synchronously. Material removal rate, machining cost, tool wear (Padhi et al., 2016), surface quality (Bhavsar et al., 2015), cutting forces (Kara and Budak, 2015), cutting fluid consumption (Jiang et al., 2015), carbon emissions (Lin et al., 2016) and energy consumption (Albertelli et al., 2016), etc., should be considered concurrently. It is impossible to optimise all objectives most at the same time (Padhi et al., 2016), because some conflicts may exist among these objectives (Tiwari et al., 2015). Optimising one objective may deteriorate another one. For example, improving surface quality increases machining time, cost and energy. In addition, it is very difficult to compare two solutions optimised in different dimensions (Zhang et al., 2015). No quantitative trade-off can be found between them. Although the MOP can be simplified as a single-objective one by weighting each objective differently, the selection of weights is arbitrary when no unified rule can be used (Li et al., 2016). The normalisation methods also come with confusing evaluation thresholds.

3 According to the Pareto rule, some non-dominated solutions can be obtained for such a MOP (Sethanan and Neungmatcha, 2016), rather than the unique optimum. Then an efficient algorithm is needed to find out the non-dominated solutions with good diversity and convergence (Asokan et al., 2003). To improve the searching ability, some multi-objective optimisation algorithm were developed on the basis of genetic algorithm (GA) (Sahali et al., 2015), ant colony optimisation (ACO), particle swarm optimisation (PSO) (Rao et al., 2016), neuro-fuzzy (Sohrabpoor et al., 2016), artificial neural network (ANN) (Maity and Mishra, 2016) and grey relational 
analysis (Kuram and Ozcelik, 2013; Patel, 2015) too. The algorithm's convergence

and the results' diversity are key issues to be addressed (Zain et al., 2011).

In summary, machining parameters' effects on residual stresses are confusing. Machining parameter selection is an MOP. Without precise mathematical models, how to take multiple objectives into account is still a problem. New optimisation algorithm should also be developed to resolve Pareto solutions with improved convergence and diversity.

To advance the study in this field, this paper puts forward a multi-objective machining parameter optimisation model. The absolute residual stress is optimised together with material removal rate and surface roughness. The multi-objective quantum cat swarm optimization (CSO) is developed to solve this problem. An example is also presented to verify the proposed model and algorithms.

The rest of this paper is organised as follows. Section 2 proposes the multi-objective machining parameter optimisation model. In Section 3, the multi-objective quantum CSO (MQCSO) is discussed in detail. An experimental study is demonstrated in Section 4, which is followed by concluding remarks in Section 5 .

\section{Multi-objective machining parameter optimisation model}

Normally, cutting speed, feed rate and cutting depth are key machining parameters that affect machining efficiency and quality. They are machining parameter optimisation variables and denoted as follows.

1 Cutting speed $(\mathrm{mm} / \mathrm{s})$ means the maximum peripheral speed of the cutter. Its value can be calculated by the following equation.

$$
v_{c}=\frac{\pi D n}{1,000 \times 60}(\mathrm{~mm} / \mathrm{s})
$$

where $D(\mathrm{~mm})$ is the diameter of the cutter and $n(\mathrm{rpm})$ is the spindle speed.

2 feed rate $f_{m}(\mathrm{~mm} / \mathrm{min})$

3 axial cutting depth $a_{p}(\mathrm{~mm})$

4 radial cutting depth $a_{e}(\mathrm{~mm})$.

Therefore, a machining parameter solution is a vector denoted as $x=\left(v_{c}, f_{m}, a_{p}, a_{e}\right)$.

Optimised machining parameters improve efficiency and better surface integrity. Here, the cutting time consumption, surface roughness and absolute residual stress are alternative approaches to these goals. They are objectives of the machining parameter optimisation problem.

1 The material removal rate (MRR) is defined as the removed material volume divided by the machining time. It can be calculated by the following equation.

$$
Q=\frac{f_{m} \cdot a_{p} \cdot a_{e}}{1,000}\left(\mathrm{~cm}^{3} / \mathrm{min}\right)
$$


Obviously, the more MRR is, the more machining efficiency will be. To transform the maximum problem to a minimum problem, the cutting time consumption of $1 \mathrm{~cm}^{3}$ material is used. It equals to the reciprocal of $Q$ and is denoted by $f_{1}(x)$ as follow.

$$
f_{1}(x)=\frac{1}{Q}=\frac{1,000}{f_{m} \cdot a_{p} \cdot a_{e}}\left(\mathrm{~min} / \mathrm{cm}^{3}\right)
$$

\section{Surface roughness}

As an important surface integrity factor, surface roughness is very important due to its effects on friction, stress, corrosion resistance, etc. Here, the minimum surface roughness $R_{a}$ is regarded as the second objective and denoted by $f_{2}(x)$. Machining parameters' effects on surface roughness are very complex. When the cutting speed $v_{c}$ increases, the surface roughness is decreasing. The surface roughness is not a monotonic function of feed rate $f_{m}$ and axial cutting depth $a_{p}$. The radial cutting depth affects the surface roughness greatly. In fact, it is very difficult to build a precise mathematical model to describe the relationship between surface roughness and the machining parameters. Here, an approximation model is built on the basis of support vector machine (SVM). The cutting speed $v_{c}$, the feed rate $f_{m}$, axial cutting depth $a_{p}$ and radial cutting depth $a_{e}$ are the inputs, while the roughness $R_{a}$ is the output. Based on experimental works, the model is trained by training examples. Parameter selection is a significant issue for SVM. Limited by the length and considering this work's main contribution, this problem is not included in this paper.

3 Absolute residual stress

Residual stress is very important, especially for thin-wall parts. A large residual stress benefits fatigue life and corrosion resistance, but small residual stress improves the size stability. Here the minimum absolute residual stress $|\sigma|$ is considered.as the third objective and denoted by $f_{3}(x)$.

Normally, the residual stress is decreasing when the cutting speed $v_{c}$ rises or when the feed rate $f_{m}$ decreases. The effects of axial cutting depth $a_{p}$ and radial cutting depth $a_{e}$ on residual stress are not very dramatic. Therefore, high spindle speed and small feed rate result in small surface stress. In fact, it is very difficult to build a precise mathematical model to describe the relationship between the residual stress and machining parameters. Here, an SVM-based approximation model is built too. The machining parameters are the inputs, while the residual stress is the output.

In summary, the final optimisation objective is presented as follow.

$$
\min f(x)=\left(f_{1}(x), f_{2}(x), f_{3}(x)\right)
$$

where $f_{1}(x), f_{2}(x)$ and $f_{3}(x)$ are cutting time consumption, surface roughness and absolute residual stress respectively. However, inevitable conflicts exist among these three objectives. For example, a large radial cutting depth decreases cutting time and deteriorates the surface roughness at the same time. A large feed rate saves cutting time and increases the absolute residual stress too. As a typical MOP, it is very difficult to optimise three objectives synchronously. A balance or a trade-off among them is needed. 
In order to make the machining parameters feasible, the following constraints apply.

1 Cutting speed should be limited in a range as follow.

$$
\frac{\pi D n_{\min }}{1,000 \times 60} \leq v_{c} \leq \frac{\pi D n_{\max }}{1,000 \times 60}
$$

where $n_{\min }$ and $n_{\max }$ are the minimum and maximum spindle speeds.

Feed rate $f_{m}$ should be limited in a range as follow.

$$
f_{\min } \leq f_{m} \leq f_{\max }
$$

where $f_{\min }$ and $f_{\max }$ are the minimum and maximum feed rates.

Axial cutting depth $a_{e}$ should be limited in a range as follow.

$$
a_{p \min } \leq a_{p} \leq a_{p \max }
$$

where $a_{p \min }$ and $a_{p \max }$ are the minimum and maximum axial cutting depth.

Radial cutting depth $a_{e}$ should be limited in a range as follow.

$$
a_{e \min } \leq a_{e} \leq a_{e \max }
$$

where $a_{e \min }$ and $a_{e \max }$ are the minimum and maximum radial cutting depth.

2 The cutting power drawn from the spindle motor should not exceed the limitation.

$$
g_{1}(x)=\frac{F_{t m} v_{c}}{6 \times 10^{4}}-\eta P_{\max } \leq 0
$$

Here, $F_{t m}$ is the maximum tangential cutting force which can be decided by the empirical formula $F_{t m}=k \cdot v_{c}^{a} \cdot f_{m}^{b} \cdot a_{p}^{c} \cdot a_{e}^{d}$ (Altintas, 2012), while coefficients a, b, c, $\mathrm{d}$ and $\mathrm{k}$ can be looked up in manuals. $P_{\max }$ and $\eta$ are the maximum spindle motor power and the efficiency respectively.

The cutting torque should not exceed the limitation.

$$
g_{2}(x)=\frac{F_{t m} D}{2 \times 10^{3}}-M_{T} \leq 0
$$

where $M_{T}$ is the maximum torque of the spindle motor.

In summary, the multi-objective machining parameter model involves four variables, including the cutting speed $v_{c}$, feed rate $f_{m}$, axial cutting depth and radial cutting depth $a_{e}$. They make a vector as $x=\left(v_{c}, f_{m}, a_{p}, a_{e}\right)$. The cutting time consumption $f_{1}(x)$, surface roughness $f_{2}(x)$ and absolute residual stress $f_{3}(x)$ are the objectives. Some constraints are used to make every solution meaningful. 


\section{Multi-objective quantum CSO}

CSO imitates the behavior of cats (Orouskhani et al., 2013). Based on the unique seeking mode and tracing mode, the convergence speed of CSO is significantly better than GA, PSO, etc. (Hadi and Sabah, 2014). Quantum computation makes direct use of quantummechanical phenomena, such as superposition and entanglement, to perform operations on data. It is believed to improve the computing ability and store capacity. In this work, the quantum computation is used to improve CSO's performance. Prior to solving the machining parameter optimisation problem, the mapping relationship between elements in the problem and CSO is shown in Figure 1.

Figure 1 Mapping relationship between the problem and CSO

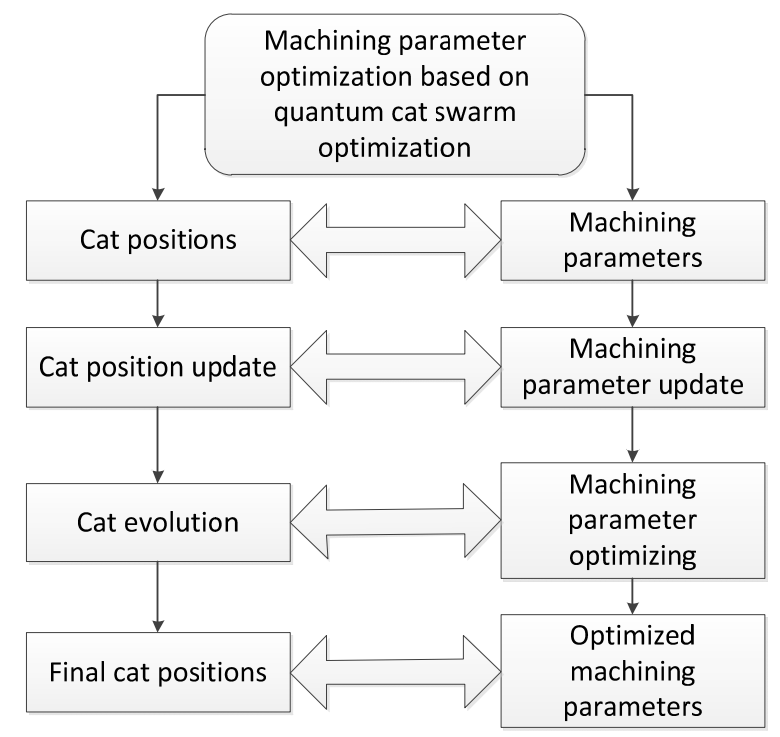

\subsection{Quantum CSO}

\subsubsection{Coding and initialisation}

Suppose the population size is nPop. A cat is mapped to a structural body that includes position (Quantum state or real number), label and fitness. Assuming the length of a quantum is $k$, the total length of all quantum is $n=4 k$. The i-th cat's position quantum is given as

$$
\left[\begin{array}{cccccccccccc}
\alpha_{i 1} & & \alpha_{i k} & \alpha_{i(k+1)} & \ldots & \alpha_{i(2 k)} & \alpha_{i(2 k+1)} & \ldots & \alpha_{i(3 k)} & \alpha_{i(3 k+1)} & & \alpha_{i(4 k)} \\
\beta_{i 1} & \cdots & \beta_{i k} & \beta_{i(k+1)} & & \beta_{i(2 k)} & \beta_{i(2 k+1)} & & \beta_{i(3 k)} & \beta_{i(3 k+1)} & & \beta_{i(4 k)}
\end{array}\right]
$$


where $\quad\left[\begin{array}{ccc}\alpha_{i 1} & \ldots & \alpha_{i k} \\ \beta_{i 1} & & \beta_{i k}\end{array}\right], \quad\left[\begin{array}{lll}\alpha_{i(k+1)} & \ldots & \alpha_{i(2 k)} \\ \beta_{i(k+1)} & & \beta_{i(2 k)}\end{array}\right], \quad\left[\begin{array}{lll}\alpha_{i(2 k+1)} & \ldots & \alpha_{i(3 k)} \\ \beta_{i(2 k+1)} & & \beta_{i(3 k)}\end{array}\right] \quad$ and $\left[\begin{array}{ccc}\alpha_{i(3 k+1)} & \ldots & \alpha_{i(4 k)} \\ \beta_{i(3 k+1)} & & \beta_{i(4 k)}\end{array}\right]$ are quantum codes of cutting speed $v_{c}$, feed rate $f_{m}$, axial cutting depth $a_{p}$ and radial depth $a_{e}$ respectively. They can be used to get real values of these variables.

With the 50/50 possibility, initial $a_{i m}(1 \leq m \leq n)$ is $1 / \sqrt{2}$ or $-1 / \sqrt{2}$ respectively. This rule applies to initial $\beta_{i m}(1 \leq m \leq)$ either. The constraint $\alpha_{i m}^{2}+\beta_{i m}^{2}=1$ applies. Then the cats' quantum states are initialised. To translate the quantum codes to binary codes, a random decimal $p$ in the range $[0,1]$ is used. If $p$ is smaller than $\left(a_{i m}\right)^{2}$, the square of the 1 st dimension's probability amplitude, $a_{i m}$ is set as 0 . Otherwise, it is 1 . The binary codes can also be translated to real values. For example, suppose the binary code of the cutting speed $v_{c}$ is $b_{\mathrm{k}} b_{k-1} \ldots b_{j} \ldots b_{2} b_{1}$, while $b_{j}(1<j<k)$ is 0 or 1 . If $v_{\mathrm{cmax}}$ and $v_{c \min }$ are the maximum and the minimum cutting speeds, the real value of cutting speed can be calculated by the following equation.

$$
v_{c}=v_{c \min }+\left(\sum_{i=j}^{k} b_{i} \cdot 2^{i-1}\right) \cdot \frac{v_{c \max }-v_{c \min }}{2^{k}-1}
$$

Similarly, the binary codes of feed rate $f_{m}$, axial cutting depth $a_{p}$ and radial cutting depth $a_{e}$ can be translated to real numbers too. The translation procedure is described by Figure 2. The real numbers are used to calculate each cat's fitness and find the optimum.

Figure 2 The translation from binary to real number

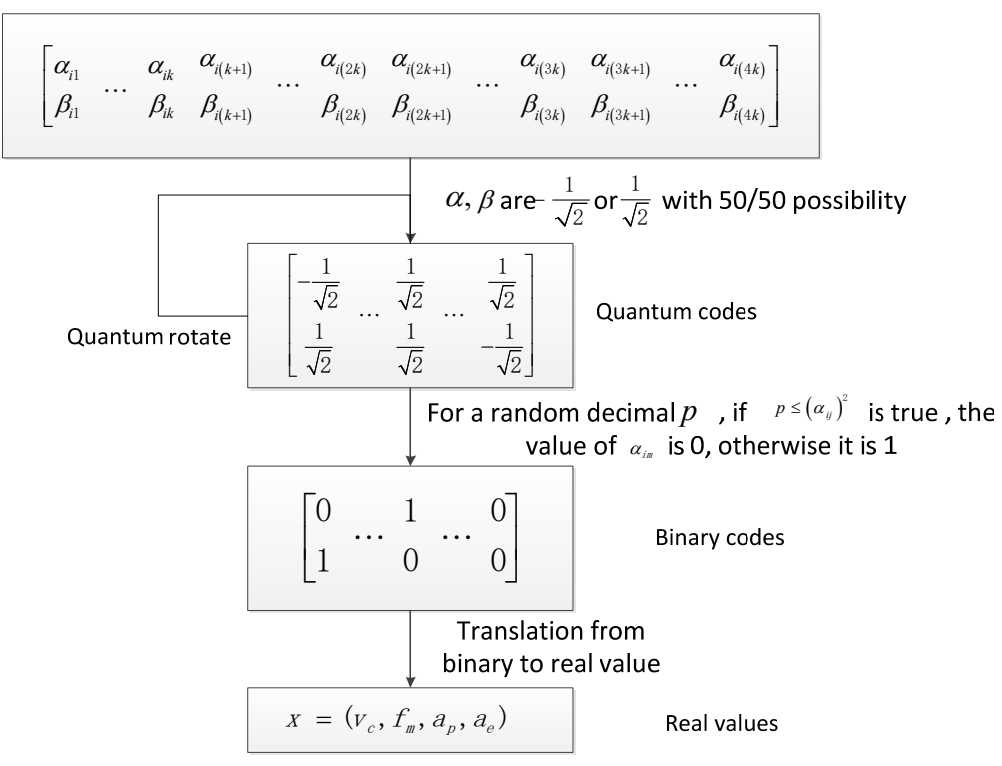




\subsubsection{Judgment of cats' modes}

The mixture ratio (MR) dictates the joining of seeking mode with tracing mode. This parameter decides the ratio of cats in tracing mode. The cat numbers in seeking mode num_seek and tracing mode num_track can be calculated by using the population nPop size and MR. Seeking mode and tracing mode describe the local searching and the global searching abilities respectively. A flag is used to identify whether a cat is in seeking mode or tracing mode. If the flag is 1 , the cat is in seeking mode. Otherwise, it is in tracing mode.

\subsubsection{Evolution of cat swarm}

The cat swarm approximates the optimum machining parameters by evolution. In each iteration, a cat in seeking mode duplicates itself $j$ times, where $j$ is the size of the seeking memory pool (SMP). According to the fitness and selecting probability, a mutated cat is selected as the result of the seeking mode.

Here, cats move via the quantum rotation. The Hadamard quantum gate $u$ is used to update the $\mathrm{i}$-th cat in the $\mathrm{t}$-th iteration by the following equation.

$$
\left[\begin{array}{c}
\alpha_{i j}^{t+1} \\
\beta_{i j}^{t+1}
\end{array}\right]=u\left(\Delta \theta_{i j}^{t+1}\right)\left[\begin{array}{l}
\alpha_{i j}^{t} \\
\beta_{i j}^{t}
\end{array}\right]=\left[\begin{array}{cc}
\cos \left(\Delta \theta_{i j}^{t+1}\right) & -\sin \left(\Delta \theta_{i j}^{t+1}\right) \\
\sin \left(\Delta \theta_{i j}^{t+1}\right) & \cos \left(\Delta \theta_{i j}^{t+1}\right)
\end{array}\right]\left[\begin{array}{c}
\alpha_{i j}^{t} \\
\beta_{i j}^{t}
\end{array}\right]
$$

where $\Delta \theta_{i j}^{t+1}(1 \leq i \leq n P o p, 1 \leq j \leq n)$ is a qubit angle increment of cat $i$ in the $\mathrm{t}+1$ iteration. The Hadamard quantum gate is shown in Figure 3.

Figure 3 The hadamard quantum gate

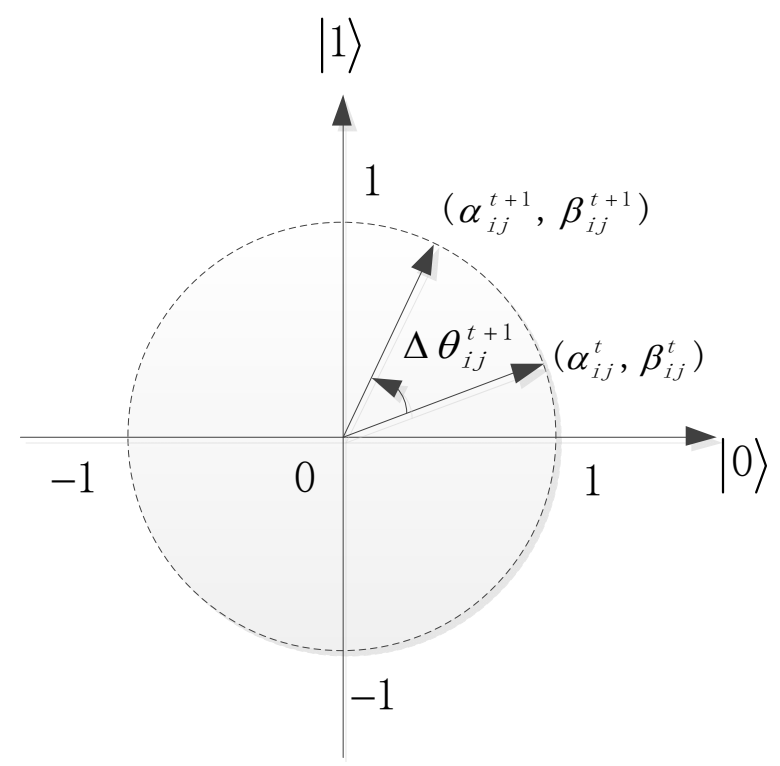

In seeking mode, the i-th cat's position is updated on the basis of the qubit angle' wave in a small range as 


$$
\Delta \theta_{i j}^{t+1}=c_{1} \pi \cdot \text { rand }
$$

where $c_{1}$ is a constant in the range $(0,1)$.

In tracing mode, the qubit angle increment of cat $i$ in iteration $j$ is

$$
\Delta \theta_{i j}^{t+1}=\Delta \theta_{i j}^{t}+c_{2} \cdot \operatorname{rand} \cdot\left(\theta_{g j}-\theta_{i j}\right) 1 \leq i \leq n P o p 1 \leq j \leq n
$$

where $c_{2}$ is a constant, $\theta_{g i}$ is the possibility angle of the global optimum cat, $\theta_{i j}$ is the possibility angle of the cat. Initially, $\left(\theta_{g i}-\theta_{i j}\right) \in[-\pi, \pi]$ is true.

The population evolution finishes when every cat completes the seeking mode and the tracing mode. The machining parameters are updated either. The global optimum can be found if each cat's fitness is calculated. The algorithm ends when the predefined iterations finish. The cat position with the maximum fitness is selected as the optimum machining parameters.

\subsection{Multi-objective quantum CSO}

In order to optimise multiple objectives synchronously, the MQCSO is studied. The optimum machining parameters are included in the Pareto front. The Pareto-layer and the niche methods are combined to solve the MOP. The penalty functions are designed according to the constraints.

1 Multi-objective optimisation method based on the Pareto-layer and niche

Suppose $f^{(u)}=\left[f_{1}^{(u)}, f_{2}^{(u)}, f_{3}^{(u)}\right]^{T}$ and $f^{(v)}=\left[f_{1}^{(v)}, f_{2}^{(v)}, f_{3}^{(v)}\right]^{T}$ are fitness vectors of solution $x^{(u)}$ and $x^{(v)}$ respectively. The domination relationship between vectors $f^{(u)}=\left[f_{1}^{(u)}, f_{2}^{(u)}, f_{3}^{(u)}\right]^{T}$ and $f^{(v)}=\left[f_{1}^{(v)}, f_{2}^{(v)}, f_{3}^{(v)}\right]^{T}$ is defined as

$\forall i=[1,2,3], f_{i}^{(u)} \leq f_{i}^{(v)} \wedge \exists j=[1,2,3], f_{j}^{(u)}<f_{j}^{(v)}$, denoted by $f^{(u)} \prec f^{(v)}$. It means that vector $f^{(u)}$ dominates vector $f^{(v)}$. Therefore, solution $x^{(u)}$ can replace solution $x^{(v)}$ if the latter is the best position in history or the global best position.

2 Niche

Based on each optimisation objective, all cats are sorted in order. Assuming $M A X_{l t}$ and $M I N_{l t}$ are the maximum and the minimum finesses of the $\mathrm{i}$-th objective in the $\mathrm{t}$-th iteration. $\operatorname{fit}_{l t}(i)$ is the fitness of the 1 -th objective of the $\mathrm{i}$-th cat in the $\mathrm{t}$-th interation. $f_{i t}(i)^{\prime}$ and $f_{i t} t_{l t}(i)^{\prime \prime}$ are respectively the fitness of two cats that are adjacent to the $\mathrm{i}$-th cat in the $\mathrm{t}$-th iteration. The niche crowding distance of the $\mathrm{i}$-th cat in the 1 -th objective is

$$
D_{l t}(i)=\frac{f i t_{l t}(i)^{\prime \prime}-f i t_{l t}(i)^{\prime}}{M A X_{l t}-M I N_{l t}}, \quad l=1,2, \ldots n .
$$

According to the niche theory, the more individuals are around, the poor diversity can be seen. Cats in sparse areas are more liable to survive than those in crowding areas because they have lower crowding distances. 
3 Selection based on Pareto and niche

Suppose $p_{(v)}$ is the v-th cat in the population $P(t)=\left\{p_{1}, p_{2}, \ldots, P_{\text {popsize }}\right\}$. The current population is $p_{u}$ and the population in the 1 -st iteration is $p_{1}$. The selection steps are listed as follows.

a Initialise the population by setting $P_{u}=P_{(t)}$ and $u=1$.

b Calculate the niche crowding distances in population $P_{u}$.

c Sort cats and find out the u-th Pareto layer $P S_{u}=\left\{\left(p_{i}, b_{i}\right)\right\}$, where $p_{i}$ is a non-dominated solution in population $P_{u}$ and $b_{i}$ is the niche crowding distance of $p_{i}$.

d Sort cats in $P S_{u}$ by $b_{i}$ in ascending order and assign rank $(v+s)$ to the s-th cat, where $s=1,2, \ldots,\left|P S_{u}\right|$.

e Calculate $\mathrm{v}=\mathrm{v}+\left|P S_{u}\right|, \mathrm{u}++, P_{u}=P_{u-1}-P S_{u-1}$. If $P_{u} \neq \varnothing$, go to Step c.

f Calculate each cat's survival possibility according to prob $\left(p_{(v)}\right)=q(1-q)^{v-1}$, $v=1,2, \ldots$, popSize, where $q$ is the possibility selection parameter.

4 Fitness function

According to equation (4), the machining time, surface roughness and absolute residual stress are going to be minimised. A calibration is needed to transform the objectives into fitness functions according to the following formula.

$$
F=k\left(-f(x)+f_{\max }+\xi\right)
$$

where $k$ is the selection possibility. It not only widens the searching range to improve the population's diversity, but also decreases the searching range to better the convergence. $\xi$ also benefits the population's diversity by providing the worse cats evolution chances.

According to the experimental results, the maximum values of three objectives are $T_{\max }=4,167 \mathrm{~min}, R_{\mathrm{amax}}=13 \mu \mathrm{m}$ and $\mid \sigma_{\max }=140 \mathrm{MP}$. The fitness functions are

$$
\begin{aligned}
& F_{1}=-f_{1}(x)+4,200 \\
& F_{2}=-100 f_{2}(x)+150 \\
& F_{3}=-\left|f_{3}(x)\right|+160
\end{aligned}
$$

The constrained optimisation problem is transformed into an unconstrained one by considering the cutting power constraint and the cutting torque constraint as penalty functions. Based on equations (9) and (10), the fitness functions are amended as follows when the penalty functions are included. Function $f_{1}(x)$ is divided by 10 to adjust its contribution to $F_{1}$.

$$
\begin{aligned}
& F_{l}=-f_{1}(x) / 10+4,200-10 g_{1}(x)-10 g_{2}(x) \\
& F_{2}=-100 f_{2}(x)+150-10 g_{1}(x)-10 g_{2}(x) \\
& F_{3}=-\left|f_{3}(x)\right|+160-10 g_{1}(x)-10 g_{2}(x)
\end{aligned}
$$




\section{Experimental study}

In order to verify the multi-objective machining parameter optimisation method, some machining experiments are done. The setup of the experimental platform is given in Table 1.

The surface roughness and residual stress testers are shown in Figures 4 and 5 respectively.

In order to make an accurate and comprehensive analysis within reasonable experiment times and complexity, an orthogonal experimental plan is designed. As Table 3 shows, cutting speed $\left(v_{c}\right)$, feed rate $\left(f_{m}\right)$, axial cutting depth $\left(a_{p}\right)$ and radial cutting depth $\left(a_{e}\right)$ are considered to make the four-factor and three-level plan. Because D10 cutting tools are used, cutting speeds $94.2 \mathrm{~m} / \mathrm{min}, 125.7 \mathrm{~m} / \mathrm{min}$ and $157.1 \mathrm{~m} / \mathrm{min}$ mean spindle speeds 3,000 r/min, 4,000 r/min and 5,000 r/min respectively. Measured surface roughness $\left(R_{a}\right)$ and residual stress $(\sigma)$ are also given in Table 2 .

Table 1 Setup of the experimental platform

\begin{tabular}{lcc}
\hline Name & Description & Memo \\
\hline Machine tool & Carver WMS1200H & 3-axis, max power 10 kw \\
Cutting tool & Eco-BRGM-4E-D10.0 & $\Phi 10 \mathrm{~mm}$ \\
Work piece & Aluminium alloy 7075 & \\
Roughness tester & TA620 & Target material: Cr, voltage: $25 \mathrm{kv}$, \\
Residual stress tester & Proto LXRD MG2000 & $\begin{array}{c}\text { current: } 30 \mathrm{~mA} \text {, exposure time: } 1 \mathrm{~s}, \\
\text { exposure number: } 10, \text { focal spot } \\
\text { diameter: 1mm, bragg angle: } 139, \text { Range } \\
\text { of flicker: } \beta \pm 25 .\end{array}$ \\
\hline
\end{tabular}

Figure 4 Roughness tester (see online version for colours)

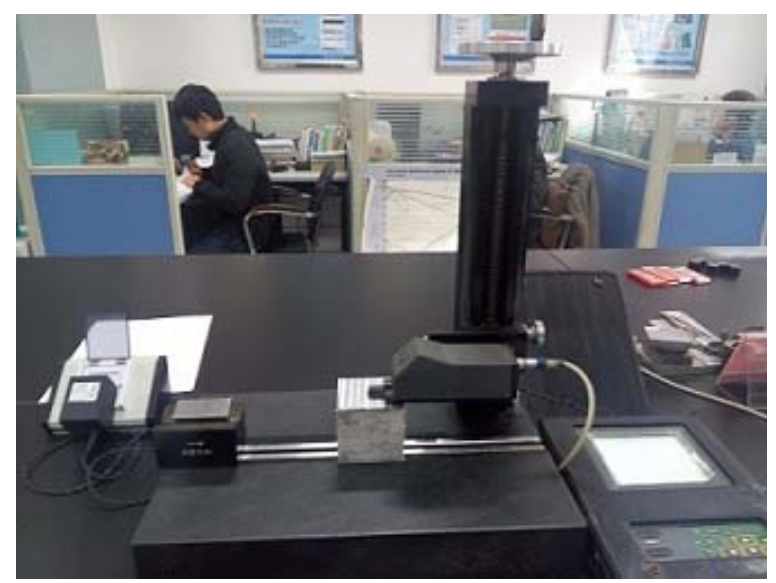


Figure 5 Residual stress tester (see online version for colours)

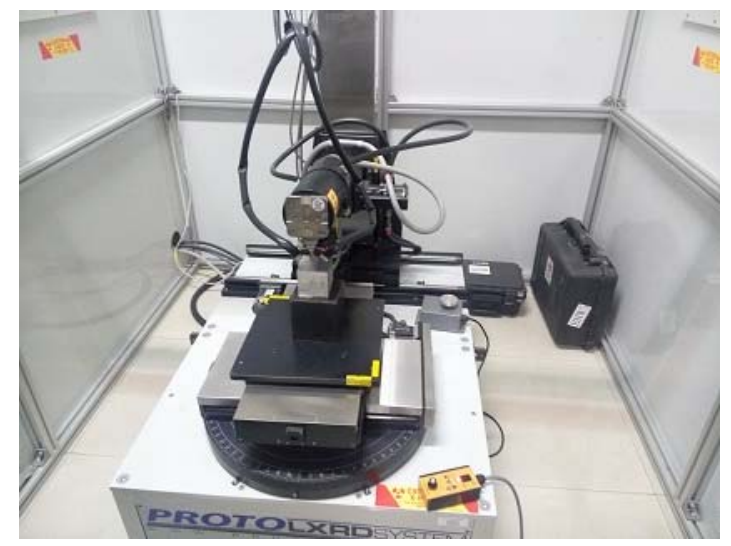

Table 2 Orthogonal experimental plan and results

\begin{tabular}{|c|c|c|c|c|c|c|}
\hline & $v_{c} / \mathrm{m} / \mathrm{min}$ & $v_{f} / \mathrm{m} / \mathrm{min}$ & $a_{p} / m m$ & $a_{e} / m m$ & $R_{a} / \mu m$ & $\sigma / M P a$ \\
\hline 01 & 94.2 & 0.4 & 0.5 & 4 & 0.788 & -63.0 \\
\hline 02 & 94.2 & 0.6 & 0.3 & 4 & 0.338 & -75.6 \\
\hline 03 & 94.2 & 0.6 & 0.5 & 2 & 0.617 & -52.4 \\
\hline 04 & 94.2 & 0.6 & 0.5 & 6 & 0.729 & -44.6 \\
\hline 05 & 94.2 & 0.6 & 0.7 & 4 & 0.614 & -38.5 \\
\hline 06 & 94.2 & 0.8 & 0.5 & 4 & 0.778 & -47.4 \\
\hline 07 & 125.7 & 0.4 & 0.3 & 4 & 0.307 & -43.8 \\
\hline 08 & 125.7 & 0.4 & 0.5 & 2 & 0.335 & -47.0 \\
\hline 09 & 125.7 & 0.4 & 0.5 & 6 & 0.461 & -46.2 \\
\hline 10 & 125.7 & 0.4 & 0.7 & 4 & 0.815 & -18.5 \\
\hline 11 & 125.7 & 0.6 & 0.3 & 2 & 0.452 & -42.0 \\
\hline 12 & 125.7 & 0.6 & 0.3 & 6 & 0.542 & -64.1 \\
\hline 13 & 125.7 & 0.6 & 0.7 & 2 & 0.417 & -53.2 \\
\hline 14 & 125.7 & 0.6 & 0.7 & 6 & 1.034 & -24.7 \\
\hline 15 & 125.7 & 0.8 & 0.3 & 4 & 0.462 & -48.5 \\
\hline 16 & 125.7 & 0.8 & 0.5 & 2 & 0.432 & -42.7 \\
\hline 17 & 125.7 & 0.8 & 0.5 & 6 & 1.047 & -43.2 \\
\hline 18 & 125.7 & 0.8 & 0.7 & 4 & 0.921 & -1.2 \\
\hline 19 & 157.1 & 0.4 & 0.5 & 4 & 0.561 & -8.3 \\
\hline 20 & 157.1 & 0.6 & 0.3 & 4 & 0.453 & -26.9 \\
\hline 21 & 157.1 & 0.6 & 0.5 & 2 & 0.606 & -16.7 \\
\hline 22 & 157.1 & 0.6 & 0.5 & 6 & 0.611 & -43.4 \\
\hline 23 & 157.1 & 0.6 & 0.7 & 4 & 0.881 & -14.4 \\
\hline 24 & 157.1 & 0.8 & 0.5 & 4 & 0.985 & -21.4 \\
\hline 25 & 125.7 & 0.6 & 0.5 & 4 & 0.560 & -16.9 \\
\hline 26 & 125.7 & 0.6 & 0.5 & 4 & 0.559 & -14.6 \\
\hline 27 & 125.7 & 0.6 & 0.5 & 4 & 0.558 & -18.9 \\
\hline
\end{tabular}


SVM-based models are built to approximate machining parameters' effects on surface roughness and absolute residual stress. In Figure 6(a), the relationship between the surface roughness and cutting speed is used to test the precision. The predicted results agree with the measured results well. Statistical results show that the root mean square (RMS) errors are no more than $9.98498 \times 10^{-2} \mu \mathrm{m}$. In Figure 6(b), the relationship between the residual stress and cutting speed is shown. The predicted results agree with the measured results well. The RMS errors are no more than $1.48356 \mathrm{MPa}$. Similar results found with other parameters verify the model's precision.

Figure 6 Results of surface roughness and residual stress by using SVM (a) surface roughness and cutting speed (b) residual stress and cutting speed (see online version for colours)

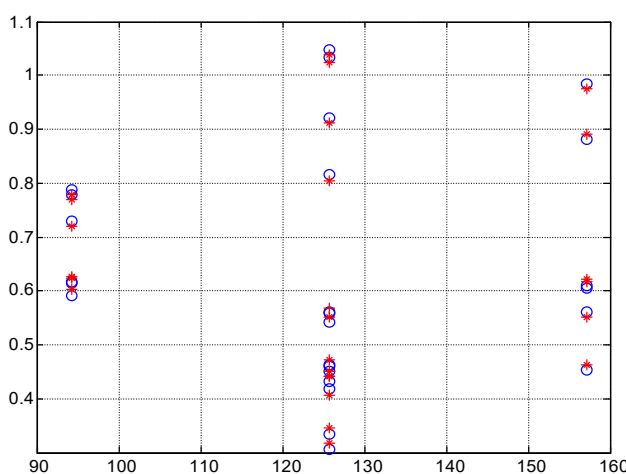

(a)

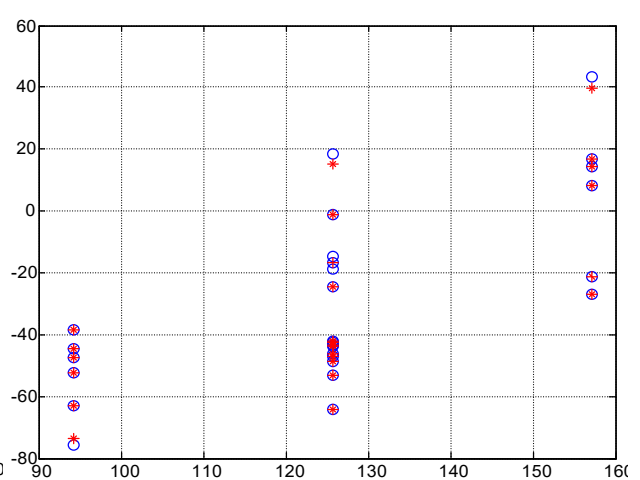

(b)

The MQCSO is implemented in MATLAB 2012. The population size is 80 and MR is 0.3. The Pareto front obtained after 100 iterations is presented in Figure 7. The algorithm shows good convergence.

Figure 7 The Pareto front after 100 iterations (see online version for colours)

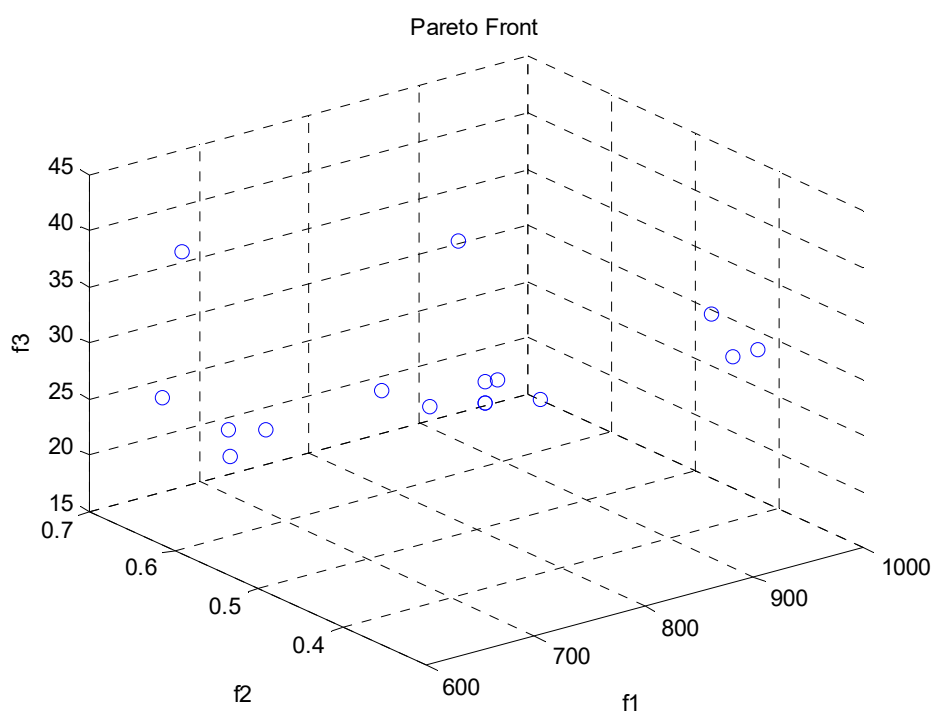


Pareto solutions in Figure 7 are listed in Table 3.

According to Figure 7, the Pareto solutions show good diversity. They can be selected as the ultimate solutions according to specific requirements. For example, to minimise the cutting time consumption, solution 1 and 5 are recommended. Their cutting time consumptions are less than $700 \mathrm{~min}$. Solution 3, 4 and 13 have better surface roughness, which is less than 0.4 . The absolute residual stresses of solution 8 and 15 are the least, $15.8 \mathrm{MPa}$. Systematically, solution 2, 7, 11 and 12 are recommended when all three objectives are balanced.

Table 3 Some Pareto solutions

\begin{tabular}{lccccccc}
\hline No. & $v_{c} / \mathrm{m} / \mathrm{min}$ & $f_{m} / \mathrm{m} / \mathrm{min}$ & $a_{p} / \mathrm{mm}$ & $a_{e} / \mathrm{mm}$ & $f_{1}(x) \mathrm{min} / \mathrm{cm}^{3}$ & $f_{2}(x) \mu m$ & $M P a$ \\
\hline 1 & 157.1 & 0.45 & 0.57 & 5.92 & 664.8 & 0.699 & 23.4 \\
2 & 157.1 & 0.51 & 0.42 & 5.98 & 780.4 & 0.497 & 41.3 \\
3 & 157.1 & 0.40 & 0.43 & 5.98 & 966.8 & 0.383 & 30.7 \\
4 & 157.1 & 0.40 & 0.43 & 5.98 & 952.8 & 0.395 & 30.0 \\
5 & 154.9 & 0.51 & 0.50 & 5.92 & 653.4 & 0.660 & 38.1 \\
6 & 157.1 & 0.44 & 0.51 & 5.00 & 892.8 & 0.611 & 21.9 \\
7 & 157.1 & 0.42 & 0.51 & 5.94 & 799.6 & 0.556 & 24.1 \\
8 & 157.1 & 0.41 & 0.59 & 4.38 & 951.5 & 0.688 & 15.8 \\
9 & 157.1 & 0.42 & 0.57 & 5.92 & 704.3 & 0.672 & 20.5 \\
10 & 157.1 & 0.42 & 0.56 & 5.91 & 723.5 & 0.652 & 20.7 \\
11 & 157.1 & 0.43 & 0.51 & 6 & 765.2 & 0.568 & 25.9 \\
12 & 157.1 & 0.40 & 0.48 & 5.98 & 853.9 & 0.494 & 25.3 \\
13 & 157.1 & 0.43 & 0.42 & 5.97 & 933.5 & 0.394 & 34.4 \\
14 & 157.1 & 0.41 & 0.59 & 4.38 & 951.5 & 0.688 & 15.8 \\
15 & 157.1 & 0.40 & 0.58 & 5.98 & 709.9 & 0.677 & 17.8 \\
16 & 157.1 & 0.44 & 0.51 & 4.93 & 904.1 & 0.611 & 21.7 \\
\hline
\end{tabular}

Solution $7,12,1,10,3$ and 13 are selected randomly to do machining experiments. The machined surfaces' roughness and residual stress are measured. The comparison between the predicted results and the measured results is shown in Table 4.

Table 4 The comparison results

\begin{tabular}{llllllll}
\hline \multirow{2}{*}{ No. } & \multicolumn{3}{c}{$f_{2}(x): R_{a} / \mu m$} & & \multicolumn{3}{c}{$f_{3}(x):|\sigma| \mathrm{MPa}$} \\
\cline { 2 - 3 } \cline { 6 - 7 } \cline { 6 - 7 } & Predicted & Measured & Error & & Predicted & Measured & Error \\
\hline 1 & 0.699 & 0.673 & $3.72 \%$ & & 23.4 & 21.7 & $7.26 \%$ \\
3 & 0.383 & 0.363 & $5.22 \%$ & & 30.7 & 29.2 & $4.89 \%$ \\
7 & 0.556 & 0.563 & $-1.26 \%$ & & 24.1 & 22.9 & $4.98 \%$ \\
10 & 0.652 & 0.637 & $1.50 \%$ & & 20.7 & 21.5 & $-3.86 \%$ \\
12 & 0.494 & 0.472 & $4.45 \%$ & & 25.3 & 24.5 & $3.16 \%$ \\
13 & 0.394 & 0.412 & $-4.57 \%$ & & 34.4 & 36.2 & $-5.23 \%$ \\
\hline
\end{tabular}


According to Table 4, the proposed machining parameter optimisation method is shown high precision. The relative roughness error and absolute residual stress error are no more than $6 \%$ and $8 \%$ respectively. The precision of proposed method is verified.

Moreover, a machining experiment is also done with a group of empirical machining parameters. The empirical cutting speed, feed rate, axial cutting depth and radial cutting depth are $v_{c}=113.04 \mathrm{~m} / \mathrm{min}, f_{m}=0.6 \mathrm{~m} / \mathrm{min}, a_{p}=0.5 \mathrm{~mm}$ and $a_{e}=4 \mathrm{~mm}$ respectively. According to equation (3), the cutting time consumption is $833.3 \mathrm{~min}$. The measured surface roughness and absolute residual stress are $R_{a}=0.638 \mu \mathrm{m}$ and $|\sigma|=64.7 \mathrm{MPa}$ respectively. Obviously, most optimised solutions have better surface roughness, while all of them have less absolute residual stress. The effectiveness of proposed method is verified.

In addition, the non-dominated sorting GA II (NSGA-II) (Li et al., 2015) is also used to solve the problem. Both NSGA-II and MQCSO run 10 times with the maximum iteration number 100 and the population size 50. Regarding to NSGA-II, the crossover rate is 0.8 and the mutation rate is 0.3 . MQCSO's MR is 0.3. The average time consumptions for 50 Pareto solutions are compared in Table 5. Obviously, MQCSO consumes less time and uses less iteration to achieve the same goal.

Table 5 Comparison between NSGA-II and MQCSO

\begin{tabular}{lcc} 
& Average time consumed & Average iteration number \\
\hline NSGA-II & $74.41 \mathrm{~s}$ & 8.1 \\
MQCSO & $65.53 \mathrm{~s}$ & 5.3 \\
\hline
\end{tabular}

Although the approach shows great potential in machining parameter optimisation, some limitations still exist. For example, machining parameters' contributions to residual stresses are still vague, because residual stresses are caused by many factors. The model may be improved to consider coolant, lubrication, time, carbon emission, and so on. Otherwise, new methods may be introduced to deal with such a complex situation either. Moreover, machining parameters should be optimised for specific part structures and machining features. More attention could be paid to weak-rigidity parts with thin-wall features.

\section{Conclusions}

In order to solve the machining parameter optimisation problem, the multi-objective machining parameter optimisation model and algorithm are proposed and shown in the following conclusions.

- The multi-objective machining parameter optimisation method optimises cutting time consumption, surface roughness and absolute residual stress systematically, while cutting power and spindle torque constraints apply.

- The SVM-based models approximate the machining parameters' effect on the surface roughness and the residual stress well.

- Improved by the quantum computation, the multi-objective CSO solves the problem with good convergence and diversity. 
- The proposed method is shown good performance in multi-objective machining parameter optimisation. Experimental study verifies the proposed method's precision and effectiveness.

In the future, more factors can be considered to improve the multi-objective machining parameter optimisation model's feasibility and practicability. For example, coolant, lubrication or carbon emission can be involved. Additional work to deal with specific thin-wall features should also be explored.

\section{Acknowledgements}

This work was supported by the Natural Science Basic Research Plan in Shaanxi Province of China [Grant Number 2013JM7001] and the National Natural Science Foundation of China [Grant Number 50805122].

\section{References}

Albertelli, P., Keshari, A. and Matta, A. (2016) 'Energy oriented multi cutting parameter optimization in face milling', Journal of Cleaner Production, Vol. 137, pp.1602-1618.

Altintas, Y. (2012) Manufacturing Automation: Metal Cutting Mechanics, Machine Tool Vibrations, and CNC Design, Cambridge University Press, New York.

Asokan, P., Saravanan, R. and Vijayakumar, K. (2003) 'Machining parameters optimisation for turning cylindrical stock into a continuous finished profile using genetic algorithm (GA) and simulated annealing (SA)', The International Journal of Advanced Manufacturing Technology, Vol. 21, No. 1, pp.1-9.

Bhavsar, S.N., Aravindan, S. and Rao, P.V. (2015) 'Investigating material removal rate and surface roughness using multi-objective optimization for focused ion beam (FIB) micro-milling of cemented carbide', Precision Engineering, Vol. 40, pp.131-138.

Hadi, I. and Sabah, M. (2014) 'Enhanced hybrid cat swarm optimization based on fitness approximation method for efficient motion estimation', International Journal of Hybrid Information Technology, Vol. 7, No. 6, pp.345-364.

Jiang, Z., Zhou, F., Zhang, H., Wang, Y. and Sutherland, J.W. (2015) 'Optimization of machining parameters considering minimum cutting fluid consumption', Journal of Cleaner Production, Vol. 108, Part A, pp.183-191.

Kara, M.E. and Budak, E. (2015) 'Optimization of turn-milling processes', Procedia CIRP, Vol. 33, pp.476-483.

Kuram, E. and Ozcelik, B. (2013) 'Multi-objective optimization using Taguchi based grey relational analysis for micro-milling of al 7,075 material with ball nose end mill', Measurement, Vol. 46, No. 6, pp.1849-1864.

Li, C., Xiao, Q., Tang, Y. and Li, L. (2016) 'A method integrating taguchi, RSM and MOPSO to CNC machining parameters optimization for energy saving', Journal of Cleaner Production, Vol. 135, pp.263-275.

Li, J., Yang, X., Ren, C., Chen, G. and Wang, Y. (2015) 'Multi-objective optimization of cutting parameters in ti-6al-4v milling process using non-dominated sorting genetic algorithm-II', The International Journal of Advanced Manufacturing Technology, Vol. 76, No. 5, pp.941-953.

Li, J.G. and Wang, S.Q. (2017) 'Distortion caused by residual stresses in machining aeronautical aluminum alloy parts: recent advances', International Journal of Advanced Manufacturing Technology, Vol. 89, Nos. 1-4, pp.997-1012. 
Lin, W., Yu, D., Zhang, C., Zhang, S., Tian, Y., Liu, S. and Luo, M. (2016) 'Multi-objective optimization of machining parameters in multi-pass turning operations for low-carbon manufacturing', Proceedings of the Institution of Mechanical Engineers, Part B: Journal of Engineering Manufacturing, pp.1-12, DOI: 10.1177/0954405416629098.

Maity, K. and Mishra, H. (2016) 'ANN modelling and elitist teaching learning approach for multi-objective optimization of $\mu$-EDM', Journal of Intelligent Manufacturing, pp.1-18, DOI: $10.1007 / \mathrm{s} 10845-016-1193-2$.

Masoudi, S., Amini, S., Saeidi, E. and Eslami-Chalander, H. (2015) 'Effect of machining-induced residual stress on the distortion of thin-walled parts', The International Journal of Advanced Manufacturing Technology, Vol. 76, No. 1, pp.597-608.

Orouskhani, M., Orouskhani, Y., Mansouri, M. and Teshnehlab, M. (2013) 'A novel cat swarm optimization algorithm for unconstrained optimization problems', International Journal of Information Technology \& Computer Science, Vol. 5, No. 11, pp.32-41.

Padhi, P.C., Mahapatra, S.S., Yadav, S.N. and Tripathy, D.K. (2016) 'Multi-objective optimization of wire electrical discharge machining (WEDM) process parameters using weighted sum genetic algorithm approach', Journal of Advanced Manufacturing Systems, Vol. 15, No. 2, pp. $85-100$.

Patel, M.T. (2015) 'Multi-objective optimization of machining parameters during turning of E250B0 of standard IS: 2,062 material using grey relation analysis', International Journal of Advanced Research in Engineering and Applied Sciences, Vol. 4, No. 6, pp.57-61.

Rao, R.V., Rai, D.P. and Balic, J. (2016) 'Multi-objective optimization of machining and micro-machining processes using non-dominated sorting teaching-learning-based optimization algorithm', Journal of Intelligent Manufacturing, pp.1-23, DOI: 10.1007/s10845-016-1210-5.

Sahali, M.A., Belaidi, I. and Serra, R. (2015) 'Efficient genetic algorithm for multi-objective robust optimization of machining parameters with taking into account uncertainties', The International Journal of Advanced Manufacturing Technology, Vol. 77, No. 1, pp.677-688.

Sethanan, K. and Neungmatcha, W. (2016) 'Multi-objective particle swarm optimization for mechanical harvester route planning of sugarcane field operations', European Journal of Operational Research, Vol. 252, No. 3, pp.969-984.

Sohrabpoor, H., Khanghah, S.P., Shahraki, S. and Teimouri, R. (2016) 'Multi-objective optimization of electrochemical machining process', The International Journal of Advanced Manufacturing Technology, Vol. 82, No. 9, pp.1683-1692.

Tiwari, A., Mandal, A. and Kumar, K. (2015) 'Multi-objective optimization of electro-chemical machining by non-dominated sorting genetic algorithm', Materials Today Proceedings, Vol. 2, Nos. 4-5, pp.2569-2575.

Zain, A.M., Haron, H. and Sharif, S. (2011) 'Optimization of process parameters in the abrasive waterjet machining using integrated SA-GA', Applied Soft Computing, Vol. 11, No. 8, pp.5350-5359.

Zhang, T., Owodunni, O. and Gao, J. (2015), 'Scenarios in multi-objective optimisation of process parameters for sustainable machining', Procedia CIRP, Vol. 26, pp.373-378.

Zhang, X., Gao, E. and Liu, C.R. (2009) 'Optimization of the process parameter of residual stresses for hard turned surfaces', Journal of Materials Processing Technology, Vol. 209, No. 9, pp.4286-4291. 